\title{
An Update on Contraception in Polycystic Ovary Syndrome
}

\author{
Seda Hanife Oguz, Bulent Okan Yildiz \\ Division of Endocrinology and Metabolism, Department of Internal Medicine, Hacettepe University School of Medicine, \\ Ankara, Turkey
}

Polycystic ovary syndrome (PCOS) is a common endocrine disorder in reproductive-aged women, characterized by hyperandrogenism, oligo/anovulation, and polycystic ovarian morphology. Combined oral contraceptives (COCs), along with lifestyle modifications, represent the first-line medical treatment for the long-term management of PCOS. Containing low doses of estrogen and different types of progestin, COCs restore menstrual cyclicity, improve hyperandrogenism, and provide additional benefits such as reducing the risk of endometrial cancer. However, potential cardiometabolic risk associated with these agents has been a concern. COCs increase the risk of venous thromboembolism (VTE), related both to the dose of estrogen and the type of progestin involved. Arterial thrombotic events related to COC use occur much less frequently, and usually not a concern for young patients. All patients diagnosed with PCOS should be carefully evaluated for cardiometabolic risk factors at baseline, before initiating a COC. Age, smoking, obesity, glucose intolerance or diabetes, hypertension, dyslipidemia, thrombophilia, and family history of VTE should be recorded. Patients should be re-assessed at consecutive visits, more closely if any baseline cardiometabolic risk factor is present. Individual risk assessment is the key in order to avoid unfavorable outcomes related to COC use in women with PCOS.

Keywords: Polycystic ovary syndrome; Contraceptives, oral; Cardiometabolic risk factors; Obesity; Diabetes mellitus; Hypertension

\section{INTRODUCTION}

Polycystic ovary syndrome (PCOS) is a very common reproductive, endocrine, and metabolic disorder characterized by hyperandrogenism, oligo/anovulation and polycystic ovarian morphology. The prevalence of the syndrome varies from $6 \%$ to $10 \%$, depending on which diagnostic criteria is used [1]. Patients usually present at a young age, during adolescence or early adulthood, with symptoms of hirsutism and/or acne, menstrual irregularity (oligo/amenorrhea), and/or infertility. Combined oral contraceptives (COCs) represent the main treatment in women not planning pregnancy in order to regulate menses and improve hyperandrogenism. However, given that PCOS is a lifelong disorder associated with certain cardiometabolic risks,

Received: 10 January 2021, Revised: 26 February 2021,

Accepted: 15 March 2021

Corresponding author: Bulent Okan Yildiz

Division of Endocrinology and Metabolism, Department of Internal Medicine, Hacettepe University School of Medicine, Hacettepe, Ankara 06100, Turkey Tel: +90-312-3051707, Fax: +90-312-3116768, E-mail: yildizbo@yahoo.com and the possible need to use COCs for a long period of time, the adverse cardiometabolic risk profile of these agents has been a concern.

The aim of this narrative review is to address the rationale, benefits, and main concerns regarding the use of contraceptives in women with PCOS in a manner that includes the most recent data.

\section{COMBINED ORAL CONTRACEPTIVES}

COCs are combinations of low-dose estrogens and progestins [2]. The first COC preparations in the 1960s containing high doses of estrogens ( $500 \mu \mathrm{g}$ of mestranol, which is equivalent of $150 \mu \mathrm{g}$ of ethinyl estradiol [EE]) [2] were abandoned due to

\section{Copyright $\odot 2021$ Korean Endocrine Society}

This is an Open Access article distributed under the terms of the Creative Commons Attribution Non-Commercial License (https://creativecommons.org/ licenses/by-nc/4.0/) which permits unrestricted non-commercial use, distribution, and reproduction in any medium, provided the original work is properly cited. 
high rates of thromboembolism, and gave way to combinations containing lower doses of estradiol $[3,4]$. The progestin component of COCs has also evolved throughout the years $[3,5]$.

Current COCs mostly contain EE, which is a modified synthetic form of estradiol, while some contain estradiol valerate (E2V), which is a natural form of estradiol (Fig. 1) [6]. Preparations having $\leq 35 \mu \mathrm{g}$ of $\mathrm{EE}$ or equivalents are called low-dose COCs $[6,7]$. Having a $17 \alpha$ ethinyl group that decelerates the rate of inactivation, EE has a stronger and prolonged effect on the liver than natural estradiol [8]. EE use leads to increased synthesis of liver proteins including lipoproteins, angiotensinogen, and estrogen-dependent clotting factors [9], which are associated with the main adverse events attributed to COCs. Combinations including lowered doses of EE [4] and more physiological forms like $\mathrm{E} 2 \mathrm{~V}$ were developed in order to reduce these adverse effects [9]. E2V imitates natural estradiol well, as a dose of 1 $\mathrm{mg}$ is equivalent to $0.76 \mathrm{mg}$ of $17 \beta$-estradiol [10].

Numerous forms of progestins are available as compounds of
COCs. Some may also have affinity for androgen, estrogen, glucocorticoid, and/or mineralocorticoid receptors in addition to the progesterone receptor [5,11]. Progestins are usually grouped into four generations, according to the time of development (Fig. 1). First-generation progestins are the initial molecules known as the norethindrone family with low potency [3]. The second-generation progestins norgestrel and levonorgestrel are more potent. The first- and second-generation progestins originate from testosterone derivatives; thus, they cause different degrees of androgenic side effects related to both their varying potencies and androgen receptor affinities [12]. However, the third-generation progestins desogestrel and gestodene are more specific to the progesterone receptor and have neglectable androgen receptor affinity; whereas, gestodene has slight mineralocorticoid activity $[5,9]$.

In the last three decades, new progestins have been developed in order to reduce androgenic side effects [11]. Trimegestone, nestorone, nomegestrol, cyproterone acetate (CPA), and chlor-

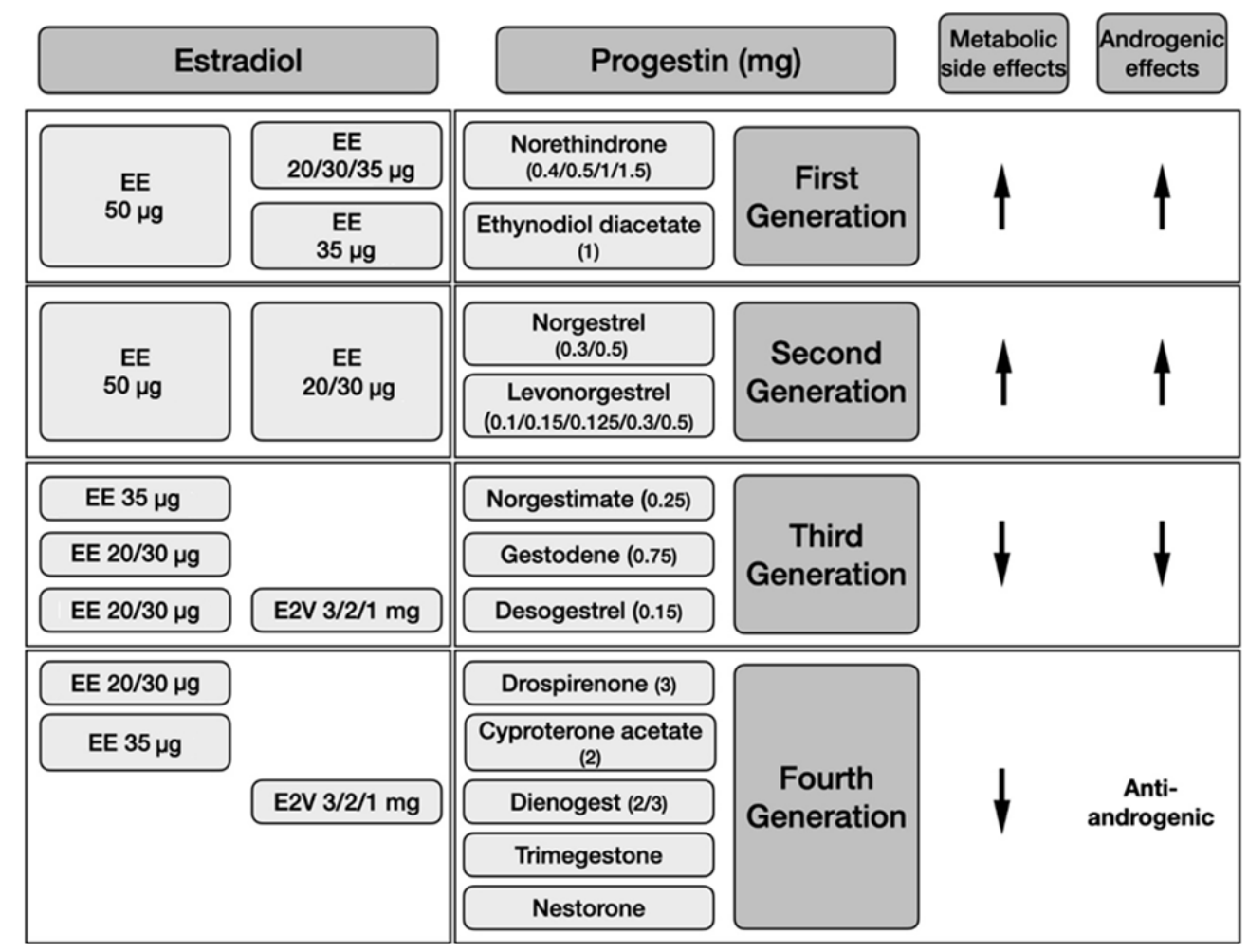

Fig. 1. Currently available combined oral contraceptive preparations according to estradiol dosage and the type of progestin, and metabolic and androgenic side effects of the progestin component. The metabolic adverse events of combined oral contraceptives are associated with both the dose of the estradiol component and the type of progestin involved. Combinations containing lowered doses of ethinyl estradiol $(\mathrm{EE} ; \leq 35 \mu \mathrm{g})$ and more physiological forms like estradiol valerate (E2V) may be chosen in order to reduce metabolic risks. First- and second-generation progestins having androgenic and metabolic side effects are usually not favored in women with polycystic ovary syndrome. Third- and fourth-generation progestins cause fewer metabolic adverse effects, and fourth-generation progestins are also anti-androgenic. Cyproterone acetate has the greatest anti-androgen activity among all progestins. 
madinone acetate are derived from progesterone, while drospirenone is a derivative of spironolactone and dienogest of testosterone [11]. Drospirenone has anti-mineralocorticoid activity antagonizing the EE-related angiotensinogen increase, in addition to its anti-androgenic effects [13]. CPA has the greatest antiandrogen activity of all progestins. The anti-androgenic activity of drospirenone and dienogest is $30 \%$ and $40 \%$ of CPA, respectively [11]. Chlormadinone acetate produces anti-androgenic effects by increasing sex hormone binding globulin (SHBG) levels and inhibiting 5- $\alpha$ reductase enzyme activity [5].

\section{MECHANISM OF ACTION}

The progestin component of COCs directly inhibits the secretion of gonadotropin-releasing hormone (GnRH) and suppresses the luteinizing hormone ( $\mathrm{LH}$ ) peak, which is essential for ovulation [14]. The absence of the LH peak causes a decrease in ovarian sensitivity to follicle-stimulating hormone (FSH), which leads to a reduction in estradiol production. Progestins also prevent sperm penetration and implantation by increasing the viscosity of cervical mucus, decreasing tubal motility, and thinning the endometrial lining [15]. The estradiol component of the drug potentiates the effect of progestin by suppressing the FSH surge in order to inhibit the selection and development of the dominant follicle, and also improves menstrual cycle control since it prevents breakthrough bleeding by maintaining endometrial proliferation [15].

Most COC regimens have a 28-day cycle: 21 days of steroid and 7 days of pill-free interval or placebo. However, the duration of a placebo has been reduced to 2 or 4 days for some COCs that contain very low doses of steroids because of concerns about contraceptive failure and/or breakthrough bleeding $[7,16,17]$.

\section{COC USE IN WOMEN WITH PCOS}

COCs, in addition to lifestyle modifications, represent the firstline treatment in most women with PCOS if fertility is not desired, in order to regulate the menstrual cycle and improve clinical signs of hyperandrogenism [18]. By suppressing LH secretion, the progestin component of COCs inhibits ovarian androgen production, whereas the estradiol component reduces serum free androgen concentrations by increasing SHBG levels $[19,20]$. Some progestins prevent androgens to bind their receptors or inhibit 5- $\alpha$ reductase enzyme activity. COCs also cause a slight decrease in adrenal androgen secretion [21].
All available guidelines and position papers, including the most recent European Society for Human Reproduction and Embriology (ESHRE)/American Society of Reproductive Medicine (ASRM)-sponsored international PCOS guideline, recommend COCs as the first-line treatment in adult women with PCOS in order to regulate menses and/or improve features of hyperandrogenism [18,22-24]. Using the lowest effective dose (20 to $30 \mu \mathrm{g}$ of EE or equivalent) is advised in order to lower metabolic risks and adverse effects [18]. It is also emphasized that a minimum duration of 6 months is required to assess treatment response regarding hirsutism [21]. The guidelines do not suggest one preparation over another [21,23], given that the current evidence is not sufficient to reveal a difference in efficacy between preparations [25].

\section{NON-CONTRACEPTIVE BENEFITS OF COCs}

Several additional benefits of COCs have been shown in the general population [26,27]. In particular, newer and lower-dose COC preparations reduce heavy menstrual bleeding. The E2V and dienogest combination has been effective in reducing heavy menstrual bleeding by $50 \%$ in about $80 \%$ of women $[16,28]$. When used continuously after surgery for endometriosis, COCs may reduce dysmenorrhea and recurrent endometriosis [29,30]. COC use has also decreased the risk of ectopic pregnancies [31], which has been attributed to the decreased risk of pelvic inflammatory disease among COC-users [32]. In cases of contraceptive failure, however, some data have indicated an increased risk of ectopic pregnancy [33]. In addition, COCs represent the second option following selective serotonin reuptake inhibitors in the treatment of physical and emotional symptoms of premenstrual dysphoric disorder [34], and combinations including drospirenone and EE might be favored [35].

In COC users, the risk of both endometrial and ovarian cancer is reduced by nearly $30 \%$ compared to non-users [36-38]. The degree of risk reduction appears to be higher with a longer duration of COC use [36,37]. While one study reported a $20 \%$ reduced colorectal cancer risk with COC use [38], no such relationship was observed in another study [36].

\section{CONTRAINDICATIONS OF COCs}

The World Health Organization (WHO) and U.S. Centers for Disease Control and Prevention (CDC) have developed evidence-based guidelines for the use of COCs. These documents, based on systematic reviews of available clinical and epidemio- 
Table 1. Absolute and Relative Contraindications of Combined Oral Contraceptive Use

Absolute contraindications

First 6 weeks postpartum, if breastfeeding

First 21 days postpartum if not breastfeeding but having other risk factors ${ }^{\mathrm{a}}$ for VTE

Age $\geq 35$ years and smoking $\geq 15$ cigarettes per day

Hypertension with BP measurements $\geq 160 / 100 \mathrm{~mm} \mathrm{Hg}$

History of current diagnosis of ischemic heart disease or history of stroke

Having multiple risk factors for cardiovascular disease

Complicated valvular heart disease

Diabetes $>20$ years duration

Diabetes with microvascular complications

Acute hepatitis

Severe cirrhosis

Liver tumors (hepatocellular adenoma or carcinoma)

Migraine with aura

Current diagnosis of cancer

History or current diagnosis of deep venous thrombosis or pulmonary embolism

Prolonged immobilization due to major surgery

Known thrombogenic mutations

Systemic lupus erythematosus with positive or un-known phospholipid antibodies

Adapted from World Health Organization [39].

VTE, venous thromboembolism; DVT, deep vein thrombosis; BP, blood pressure.

aPrevious VTE, thrombophilia, immobility, transfusion at delivery, body mass index $>30 \mathrm{~kg} / \mathrm{m}^{2}$, postpartum hemorrhage, immediately after cesarean delivery, pre-eclampsia, and smoking.

logical research, are updated regularly. The most recent versions of the WHO and CDC guidelines were published in 2015 and 2016, respectively [39,40]. The ESHRE/ASRM-sponsored international PCOS guideline suggests following the WHO guidelines for relative and absolute contraindications for COC use (Table 1) [18]. Accordingly, it is recommended that COCs containing $35 \mu \mathrm{g}$ of EE and CPA should not be used in mild cases, and should only be used to treat moderate to severe hirsutism or acne due to higher risk of venous thromboembolism (VTE). Other preparations with lower VTE risk should be preferred as the first-line therapy for purposes of contraception, regulating menses, or treating mild to moderate hirsutism [18].

\section{CONCERNS REGARDING THE USE OF COCs IN PCOS}

\section{Venous thromboembolism}

The incidence of VTE is reported to be 5 to 10 events per 10,000 woman-years in the general population [41]. COC use

\section{Relative contraindications}

Six weeks to $<6$ months postpartum, if breastfeeding First 21 days postpartum, if not breastfeeding

First 42 days postpartum, if not breastfeeding but having other risk factors ${ }^{\mathrm{a}}$ for DVT

Age $\geq 35$ years and smoking $<15$ cigarettes per day

Hypertension controlled with medication or BP measurements between 140-159/90-99 $\mathrm{mm} \mathrm{Hg}$

Dyslipidemia

\section{Symptomatic gall bladder disease}

History of cholestasis related to oral contraceptive use Using rifampicin or rifabutin

Using anticonvulsant medications

Migraine without aura

History of breast cancer cured for $\geq 5$ years can increase the incidence of VTE events up to $8-10 / 10,000$ woman-years [42], which is lower than the incidence of pregnancy-associated VTE (1.2 of every 1,000 deliveries) [43,44]. Overall, the relative risk (RR) of VTE in all kinds of COC users has been reported to be 3.5 (95\% confidence interval [CI], 2.9 to 4.3) [45], and is significantly higher during the first year of use and in women who are new to COCs [46].

The risk of VTE associated with COC use changes according to both the dose of estrogen and the type of progestin. In a population-based case-control study, in patients using different types of COC preparations, the relative thrombotic risk was 0.8 (95\% CI, 0.5 to 1.2 ) and 1.9 (95\% CI, 1.1 to 3.4) for estrogen doses of 20 and $50 \mu \mathrm{g}$ respectively, compared to the reference estrogen dose of $30 \mu \mathrm{g}$ [47]. A Cochrane Review reported the RR of VTE as 2.2 (95\% CI, 1.3 to 3.7) with $50 \mu \mathrm{g}$ of EE compared to $30 \mu \mathrm{g}$, when used in combination with levonorgestrel [45]. No difference has yet been shown between EE doses of 20 and $30 \mu \mathrm{g}(\mathrm{RR}, 1.1 ; 95 \% \mathrm{CI}, 0.7$ to 1.7$)$ [45,46]. Thus, there is no evidence showing that reducing the dose of EE below $35 \mu \mathrm{g}$ 
Table 2. Risk of Venous Thromboembolism among Combined Oral Contraceptive Users According to the Type of Progestin

\begin{tabular}{lc}
\hline Variable & VTE risk, RR $(95 \%$ CI $)$ \\
\hline Second-generation & 1 \\
Levonorgestrel & $1.14(0.94-1.32)^{\mathrm{a}}$ \\
Third-generation & $1.67(1.32-2.10)^{\mathrm{a}}$ \\
Norgestimate & $1.27(1.15-1.4)^{\mathrm{b}}$ \\
Gestodene & $1.83(1.55-2.13)^{\mathrm{a}}$ \\
& $1.46(1.33-1.59)^{\mathrm{b}}$ \\
Desogestrel & \\
Fourth-generation & $1.58(1.12-2.14)^{\mathrm{a}}$ \\
Drospirenone & $1.40(1.26-1.56)^{\mathrm{b}}$ \\
& $2.04(1.55-2.49)^{\mathrm{a}}$ \\
Cyproterone acetate & $1.29(1.12-1.49)^{\mathrm{b}}$ \\
& $1.46(0.57-5.41)^{\mathrm{a}}$ \\
\hline
\end{tabular}

VTE, venous thromboembolism; RR, relative risk; CI, confidence interval.

The estimated RR for VTE for 1 year of combined oral contraceptive use was provided from the meta-analyses by ${ }^{\mathrm{a} D r a g o m a n}$ et al. [42] and bedingen et al. [48], respectively, in comparison to levonorgestrel. Data on VTE risk were obtained from the general population, and the absolute risk of VTE is low (8-10/10,000 woman-years).

in COCs decreases the risk of VTE [46].

A recent meta-analysis revealed increased VTE risk with combinations containing CPA (RR, 2.04; 95\% CI, 1.55 to 2.49), desogestrel (RR, 1.55; 95\% CI, 1.55 to 2.13), drospirenone (RR, 1.58 ; $95 \%$ CI, 1.12 to 2.14 ), and gestodene (RR, $1.67 ; 95 \% \mathrm{CI}$, 1.32 to 2.10 ) compared to levonorgestrel. The RR of VTE was similar between levonorgestrel and norgestimate (RR, 1.14; $95 \% \mathrm{CI}, 0.94$ to 1.32 ) and dienogest (RR, 1.46; $95 \% \mathrm{CI}, 0.57$ to 5.41), respectively [42]. Another meta-analysis also indicated a considerably increased risk of VTE for desogestrel, drospirenone, gestodene, and CPA compared to levonorgestrel, when combined with EE (30 to $40 \mu \mathrm{g}$ ) (Table 2). No increased risk of VTE was reported for gestodene/EE $20 \mu \mathrm{g}$ [48] and dienogest/ E2V [49] combinations.

COCs significantly increase the risk of VTE, especially if the baseline VTE risk is high. The main risk factors for VTE in COC users are prolonged immobilization, smoking, age over 35 years, obesity, a personal or family history of VTE or inherited thrombophilia, antiphospholipid syndrome, active systemic lupus erythematosus, and current cancer diagnosis [46]. In addition, PCOS itself has been reported to be a prothrombotic con- dition, as studies revealed disruptions in fibrinolysis in women with PCOS [50,51]. An analysis of a commercial database from United States reported a 2 to 3-fold increased risk of VTE in women with PCOS compared to the general population between 2003 and 2008, which decreased by $20 \%$ with oral contraceptive use in PCOS patients [52]. A recent meta-analysis of five observational studies between 2004 and 2018 reported a 1.5 to 2-fold increased risk for VTE in women with PCOS, not entirely attributable to high incidence of COC use or excess weight in these patients [53].

In the absence of other risk factors for VTE, it remains controversial whether COCs further increase the absolute risk of VTE in women with PCOS compared to healthy women [54]. Of interest, although one study suggested that the risk of VTE was even lower in women with PCOS using COCs than in those not using COCs [52], that may have been due to prescription bias, and might suggest that careful evaluation of PCOS patients for risk factors before initiating COCs would be often enough to prevent VTEs [55]. Nevertheless, given that COCs containing CPA pose a significant risk of VTE, it is recommended to avoid prescribing these preparations for PCOS patients with mild hyperandrogenism [18].

Overall, available data indicates that the use of COCs increases the risk of VTE in the general population. However, most studies addressing the topic have not evaluated all potential confounding factors, and may be subject to bias. It remains controversial whether COCs create an additional risk of VTE in PCOS, which is already a prothrombotic condition. It is important to keep in mind that the COC-related absolute risk for VTE is low in young healthy women with or without PCOS; and that careful assessments of patients before prescribing COCs would be the best measure to prevent VTEs in women with PCOS.

\section{Myocardial infarction and stroke}

A Cochrane meta-analysis indicated a 1.6-fold increased risk of overall arterial thrombosis in premenopausal women (18 to 50 years of age) using COCs [56]. The RRs appeared to be similar for myocardial infarction (MI; 1.6; 95\% CI, 1.2 to 2.1) and ischemic stroke $(1.7 ; 95 \%$ CI, 1.5 to 1.9$)$. Higher EE doses were associated with increased risk, while the type of progestin did not appear to have an impact [56]. Unlike VTE, the risk of arterial thrombosis does not diminish over time with COC use [57]. As expected, the rates of $\mathrm{MI}$ and ischemic stroke in COC users increase with age. Arterial thrombosis related to COC use occurs less often than VTE, especially at younger ages. It is estimated that there are two arterial thrombotic events attributable to $\mathrm{COC}$ 
use per 10,000 women per year [57].

Many women with PCOS already carry some risk factors for cardiovascular disease, such as obesity, insulin resistance, dyslipidemia, dysfibrinolysis, and hypertension, which may be poorly affected by COC use. However, the evidence remains controversial regarding whether there is an absolute increase in risk for MI, stroke, cardiovascular death, or coronary heart disease in women with PCOS in the long-term [58,59], including peri- and postmenopausal periods [60-63]. Likewise, the risk of stroke-related mortality seems not to increase in PCOS [64]. Even so, a significant amount of data on the subject has been provided from retrospective or cross-sectional studies with small sample sizes, without taking several confounding factors into account, such as COC use [58,59].

Several hypotheses have been suggested to explain the absence of unfavorable cardiovascular outcomes in PCOS despite the abundance of risk factors [60]; however, none has yet been confirmed. The effect of long-term COC use on future cardiovascular events remains unclear [65]. A study of 672 postmenopausal women with at least one cardiovascular risk factor revealed that prior use of COCs, regardless of duration, was associated with lower coronary artery disease severity scores as assessed by coronary angiography. The statistical relationship remained significant after adjusting for other cardiovascular risk factors including age, diabetes, dyslipidemia, smoking, and hypertension [66].

The long-term effects of COC use on cardiovascular outcomes in women with PCOS remains to be clarified, especially during the later years of life. Until then, it would be best to evaluate patients for cardiovascular risk factors before starting COCs and during follow-up, according to currently available guidelines [18,22].

\section{Abnormalities in glucose metabolism and diabetes}

PCOS is associated with higher rates of impaired glucose tolerance (IGT) and type 2 diabetes mellitus (T2DM). Although the degree of the risk is related to race, obesity and increasing age, normal-weight women with PCOS also have a higher risk of developing IGT and T2DM than age- and body mass index (BMI)-matched healthy controls. While IGT is the leading form of glucose intolerance at younger ages, patients generally start to develop diabetes throughout their peri- or postmenopausal years [58]. Based on limited data, the risk of developing diabetes in women with PCOS during later years of life appears to be related to higher BMI, abdominal obesity, and insulin resistance, but not hyperandrogenism $[67,68]$.
In population studies conducted on healthy premenopausal women, COCs seem to have no unfavorable effect on glucose metabolism [69,70]. Likewise, two meta-analyses of PCOS patients revealed no changes in levels of fasting plasma glucose, fasting insulin, and homeostatic model assessment for insulin resistance (HOMA-IR) scores related to COC use for up to a year. However, most of the outcomes revealed significant heterogeneity in both meta-analyses, and majority of the included studies had inherent limitations [71,72]. Considering that the differences in types of COCs were not able to explain the heterogeneity, it was suggested that COCs were not different from each other in terms of glycemic outcomes [71].

Although current data do not indicate any adverse effects of COCs on glucose metabolism, there is still need for high-quality long-term prospective studies with large sample sizes. It should be emphasized that there is no hard evidence to justify avoidance of COC use in PCOS patients with metabolic disturbances, or to recommend one COC over another [24]. For each patient, individual characteristics such as age, ethnicity, BMI, and family history of diabetes should be considered when determining the risk of diabetes [6]. It is very important to encourage all women with PCOS to maintain a healthy lifestyle and diet. An oral glucose tolerance test with $75 \mathrm{~g}$ of glucose should be performed at baseline and regularly thereafter, especially if obesity and/or other risk factors are present (Fig. 2).

\section{Other cardiometabolic effects}

The prevalence of metabolic syndrome and its individual components is increased in women with PCOS [73]. In a large population, we reported $12.5 \%, 10.3 \%$, and $10.0 \%$ prevalence rates of metabolic syndrome in unselected women with PCOS according to the criteria of the National Institutes of Health, Rotterdam, and the Androgen Excess and PCOS Society, respectively, suggesting at least 2-fold increased risk compared to general population [74]. This increase is similar to the risk reported in women with PCOS presenting to the clinic [75].

Hyperandrogenism contributes to insulin resistance through various pathways [76], and improving hyperandrogenism may provide some beneficial metabolic effects. Accordingly, the anti-androgenic effects of COCs may improve metabolic parameters [77]. Along with lifestyle modifications, treatment with COCs may improve insulin resistance, serum total cholesterol levels, and serum low-density lipoprotein (LDL) cholesterol levels in patients with PCOS [77,78]. However, COCs may lead to an increase in serum levels of triglycerides, LDL cholesterol, and high-density lipoprotein (HDL) cholesterol due to their ef- 


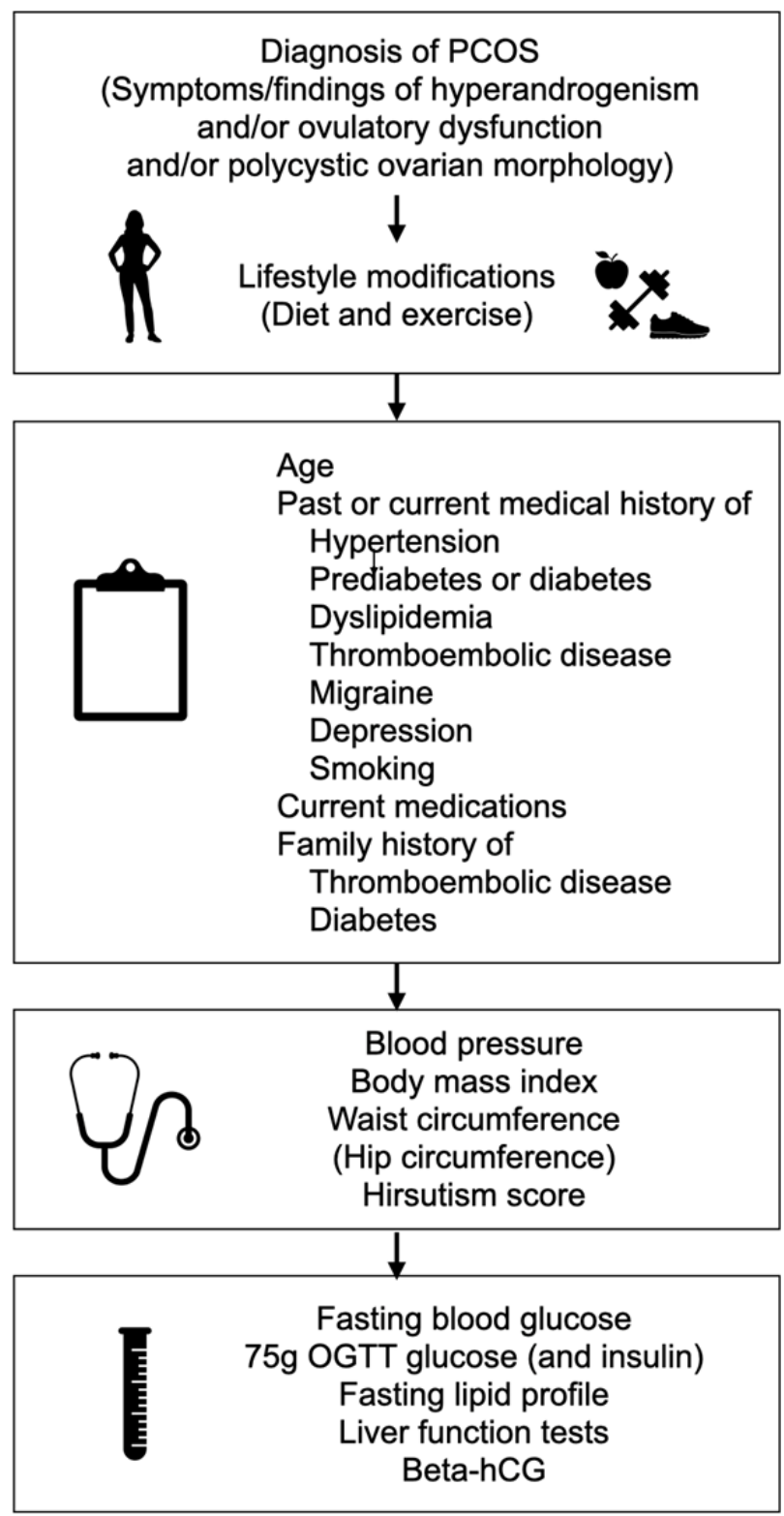

Fig. 2. Assessment algorithm in women with polycystic ovary syndrome (PCOS) before prescribing a combined oral contraceptive. OGTT, oral glucose tolerance test; hCG, human chorionic gonadotropin.

fects on the liver $[9,79]$. Some studies revealed better outcomes with drospirenone regarding LDL levels [80,81], whereas others did not detect any differences between different types of progestins involved [79,82]. Currently, there is not sufficient evidence to favor one combination over another.

An increase in appetite and weight gain associated with $\mathrm{COC}$ use are concerns commonly expressed by patients with PCOS [6]. An earlier study suggested a suppression in cholecystokinin levels with the use of EE/desogestrel combination in healthy lean women [83]. However, in lean women with PCOS, shortterm use of combined EE/drospirenone did not alter the levels of the satiety hormones cholecystokinin and peptide YY and the hunger hormone ghrelin [84]. Moreover, EE/drospirenone treatment was associated with reduced hunger and increased satiety after meals, and reduced craving for sweets in lean hyperandrogenic bulimic women, without a significant change in associated gut hormone and peptide levels [85]. Although the effect of COCs on appetite may differ among patients and possibly according to the type of progestin, the current data suggest no major association.

Studies in the general population have not reported significant weight gain with COCs [86], even in long-term analyses [87]. However, COC use might result in the redistribution of body fat. Studying 28 hyperandrogenic lean women with PCOS, we reported a significant increase in total and truncal fat percentage after 6 months of EE/drospirenone use, despite no change in clinical anthropometric measures including BMI, waist circumference, or the waist-to-hip ratio (WHR) [88]. Another observational study of hyperandrogenic and insulin-resistant PCOS patients, including 16 patients on COC treatment (EE/CPA, EE/ gestodene, or EE/desogestrel) and 21 patients who had never used COCs found that waist circumference and WHR decreased in the COC arm, whereas BMI, waist circumference, and WHR remained unchanged in the non-COC arm. The mean duration of follow-up was 10 years (range, 12 to 180 months), and the mean ages at the beginning and the end of the study were 18 and 29 years in the COC group, and 21 and 31 years in the nonCOC group, respectively [89].

A retrospective study including 1,297 women with PCOS with a mean age of 28 years categorized the subjects as current users, who had been using COCs for at least the last three months $(n=76)$, and ever users, while the remaining 203 subjects who had never used a COC were taken as the reference group [90]. Ever users were further divided into five subgroups according to duration of COC use. No differences were found between groups or subgroups regarding anthropometric measurements, lipid profile, or HOMA-IR scores [90]. Ibanez et al. [91] reported increased carotid intima media thickness, visceral obesity, and higher levels of serum high-sensitivity C-reactive protein (hsCRP) levels with the use of COC containing CPA in comparison with low-dose insulin-sensitizer medications in adolescent PCOS patients. Likewise, we have found increased hsCRP and homocysteine levels in young lean PCOS patients using a drospirenone-containing $\mathrm{COC}$ with spironolactone, compared to age- and BMI-matched controls [92]. 
COCs could potentially cause an increase in blood pressure (BP) due to the increased production of angiotensinogen in the liver induced by the estradiol component [9]. This increase in BP could be less significant with preparations containing lowdose estradiol, but may still be a concern for patients who already have hypertension. Accordingly, BP should be checked in all women with PCOS prior to starting a COC, since a number of patients may be unaware of having hypertension [93].

Taken together, studies do not indicate a worsened cardiometabolic risk profile with the use of COCs in PCOS, although good-quality evidence is still needed from long-term studies including greater number of patients and comparing different combinations of COCs. The association of COC use with increased inflammatory parameters in young PCOS patients requires confirmation.

\section{Psychological impact}

Studies in the general population suggest that COC use might occasionally be associated with mood alterations, particularly in younger patients and those with previous mental health problems [94-97]. In PCOS, the risk of having symptoms related to depression (odds ratio [OR], 3.78; 95\% CI, 3.03 to 4.72) or anxiety (OR, 5.62; 95\% CI, 3.22 to 9.80) is already increased [98]. Depression appears to be linked with cardiometabolic features of PCOS, including obesity, insulin resistance, and dyslipidemia [99]. However, contradictory results have been reported regarding how COCs affect psychological symptoms in women with PCOS [96,99].

A large Danish cohort study including over one million women between 15 and 34 years of age with a mean follow-up duration of 6.4 years revealed higher rates of starting anti-depressant treatment among hormonal contraceptive users (55\% of the study population) [100]. Compared to non-users, the RR for starting an anti-depressant was 1.22 (95\% CI, 1.22 to 1.25$)$ in COC users. The risk was associated with a longer duration of COC use, but not with smoking or BMI. Notably, the risk remained significant after adjustment for diagnosis of PCOS. The RR for starting an anti-depressant among adolescents using contraceptives was even higher (RR, 1.8; 95\% CI, 1.75 to 1.84 ) [94]. In a large Swedish cohort, the use of both COC (hazard ratio [HR], 1.4; 95\% CI, 1.22 to 1.60 ) and progestin-only pills (HR, 2.18; 95\% CI, 1.81 to 2.62) was associated with suicidal behavior. The risk was highest at the first month after initiation and declined with longer duration of use, but remained high for at least 1 year [100].

The limited available data do not suggest an increased risk of depression with COC use in PCOS patients. In a prospective study assessing the effect of 6 months of COC use on 36 women with PCOS, depression scores did not show a significant difference before and after treatment; contrarily, PCOS-specific quality of life scores improved as hirsutism regressed [99]. A secondary analysis of the OWL-PCOS study also revealed that COCs positively affected symptoms of depression and anxiety as a result of improved hirsutism and weight loss [101].

Recent international guidelines emphasize the high prevalence of anxiety and depressive symptoms in adult patients with PCOS [18]. It would be helpful to consider the potential effects of COCs on emotional well-being in women with PCOS, along with risk factors such as obesity, infertility, and hirsutism.

\section{STARTING COC TREATMENT AND PATIENT FOLLOW-UP}

The assessment algorithm before starting COC treatment in PCOS patients is given in Fig. 2 [6]. The medical history must cover all relevant information on cardiometabolic risk factors including hypertension, dyslipidemia, smoking, glucose intolerance, and diabetes; as well as diagnosis of migraine and depression. Furthermore, information must be elicited on patients' own and family history of thromboembolic events and diabetes.

Baseline BMI, waist circumference, BP measurements, and hirsutism score should be recorded, but performing breast, pelvic, and genital examinations or cervical cytology is not routinely recommended. In order to assess baseline cardiometabolic risk, fasting blood glucose, a 75-g standard 2-hour oral glucose tolerance test and fasting lipid profile need to be performed. Liver function tests and beta-human chorionic gonadotropin should also be ordered.

Patients should be informed about common side effects, such as gastrointestinal complaints, breast tenderness, headache, and mood changes, which would possibly diminish or disappear in months. Weight gain is usually a main concern expressed by patients. Although studies have not shown any association between COC use and weight gain [86], many women complain about some weight gain during COC use [6]. Thus, regular follow-up of anthropometric measures, including BMI and waist circumference, is required. It must be emphasized to patients with hirsutism that it will take at least 6 months of treatment until any clinical improvement can be observed [102].

Patients need to be informed about serious but rare adverse effects of COCs. Red flag signs or symptoms of VTE or arterial thrombosis such as leg swelling, new onset severe headache or 
visual disturbances, chest pain, numbness, or muscle weakness require immediate medical attention. However, it should be kept in mind that in the absence of other risk factors, COC-related VTE or arterial thrombotic events are very rare. Routine screening for thrombophilia is not recommended, but may be considered in high-risk patients.

The ideal time to start COCs is on the 1st to 5th days of the menstrual cycle. They can be started at any other time once it is confirmed that the patient is not pregnant.

Available guidelines recommend an individualized approach for follow-up and provide clinical practice points based on expert opinion $[6,18,103]$. The first follow-up visit should be scheduled for after 3 months of treatment. BMI, waist circumference, and BP should be recorded. Laboratory tests would be performed according to the baseline results and each patient's baseline condition. If there are no side effects and the patient is comfortable, COC treatment is continued. Hirsutism is reassessed after 6 months of treatment. Subsequent follow-up visits would be scheduled every 6 months $[6,18,103]$.

\section{PROGESTIN-ONLY CONTRACEPTIVES}

Progestin-only contraceptives could be used for contraception in women who have contraindications or intolerance for COCs, such as a high-risk or history of venous or arterial thrombotic conditions [104]. While the majority of these contraceptive methods appear not to have any adverse cardiometabolic effects in healthy female population [105-110], contradictory data have been reported [106,111]; however, very few studies assessed the use of these methods in PCOS patients. Depot medroxyprogesterone acetate (DMPA) is considered to have a more unfavorable effect on cardiometabolic parameters than other progestinonly contraceptive methods [112].

\section{Progestin-only pills}

Progestin-only pills, or mini-pills, containing low daily doses of progestins (e.g., levonorgestrel $30 \mu \mathrm{g}$, norethindrone $35 \mu \mathrm{g}$, desogestrel $75 \mu \mathrm{g}$ ) need to be used continuously [113], except for the drospirenone-only pill, which is taken daily for 24 days followed by 4 days of placebo [114]. In continuous use, higher rates of breakthrough bleeding than that of COCs were reported [115]. Alternatively, progestins such as micronized natural progesterone or oral medroxyprogesterone acetate (MPA) may be given in short cycles in amenorrheic PCOS patients in order to protect the endometrium from the hyperplasic effect of unopposed estrogen exposure [116]. While the only absolute contra- indication for progestin-only pills is current breast cancer, an alternative contraceptive method would be preferred in patients with severe cirrhosis, hepatocellular adenoma/carcinoma, or an acute or past history of ischemic stroke or ischemic coronary event [112].

In lean hyperandrogenic women with PCOS, treatment with oral MPA and micronized progesterone for 10 days were both associated with reductions in fasting insulin levels, HOMA-IR scores, HDL, LDL, and total cholesterol concentrations in a small-sized prospective randomized study, but the reductions were only significant in the MPA group [117]. Another prospective study including young overweight women with PCOS reported an immediate decrease in HOMA-IR score after 7 days of micronized progesterone use, which returned to baseline after withdrawal bleeding [118]. Overall, current data are insufficient regarding the metabolic impacts of oral MPA and micronized progesterone in women with PCOS. Although no studies to date have evaluated the confined effects of mini-pills in PCOS patients in this regard, they seem not to affect cardiometabolic parameters in healthy women [108,119].

\section{Depot medroxyprogesterone acetate}

DMPA is an injectable progestin-only contraceptive administered every 3 months [40]. The contraindications and warnings specified for progestin-containing pills also apply to DMPA. In addition, DMPA should be avoided in women with complicated diabetes [112]. According to a prospective case-control study, 30 months of DMPA usage in healthy young women significantly increased body weight, which was entirely attributed to increased total fat mass with central deposition [120]. Decreased bone mineral density is another adverse effect of DMPA [121]. There is a lack of data regarding the use of DMPA in PCOS patients.

\section{Etonogestrel}

Etonogestrel is the active metabolite of desogestrel. Studies in healthy women using etonogestrel implants have not reported any adverse metabolic effects [109,110]. However, a small study in patients with PCOS suggested increased insulin resistance with a 6-month duration of etonogestrel implant usage [122].

Levonorgestrel-releasing intrauterine contraceptive device Levonorgestrel-releasing intrauterine device (LNG-IUD), in a central reservoir, contains $52 \mathrm{mg}$ of levonorgestrel, with a daily released dose of $20 \mu \mathrm{g}$. With a 5-year duration of recommended 
use [123], it is one of the most cost-effective reversible contraceptive methods [124]. LNG-IUD has anti-proliferative effects on the endometrium, and has been shown to be superior to nonintrauterine progestins at reversing endometrial hyperplasia [125]. Although declining plasma levonorgestrel concentrations were reported with a longer duration of use and higher BMI [126], it was still more effective than oral progestins in the treatment of endometrial hyperplasia in obese subjects [127]. LNGIUD has also been reported to reverse endometrial hyperplasia [128] and early endometrial carcinoma $[129,130]$ in patients with PCOS. LNG-IUD has also been superior to both non-intrauterine progestins [131] and COCs [132] in reducing heavy menstrual bleeding. LNG-IUD is also effective in the treatment of endometriosis and associated pelvic pain [133]. Its use is absolutely contraindicated in cases of unexplained vaginal bleeding, severe cervical or intrauterine infection or sepsis, current diagnosis of breast cancer, and distorted uterine cavity [40]. There is also a risk of uterine perforation [134].

LNG-IUD usage was not associated with adverse cardiometabolic effects in healthy women [106,107], except a mild increase in fasting plasma glucose levels from baseline [106]. In an observational prospective controlled study including nonobese patients with PCOS, 6 months of LNG-IUD use improved hyperandrogenemia, but increased waist circumference and fasting plasma glucose measurements, whereas decreased LDL and total cholesterol concentrations compared to baseline [135].

\section{CONCLUSIONS}

All women with PCOS should be encouraged to maintain a healthy lifestyle. COCs are the primary choice of treatment in PCOS patients in order to achieve the goals of regulating menses and improving signs/symptoms of hyperandrogenism. Current guidelines do not provide a basis for preferring one $\mathrm{COC}$ over another, but advise using formulations containing lower estrogen doses.

COCs increase the RR of venous and arterial thrombotic events, however, the absolute risk is low in young healthy nonsmoking women. A careful medical history and ruling out additional risk factors before starting treatment would almost fully eliminate the risk of thrombosis. Although long-term observational studies are lacking, there is no evidence showing adverse cardiometabolic outcomes with short-term use of COCs. Nevertheless, patients who are obese, or who already have IGT or other cardiometabolic risk factors, should be monitored more closely. Progestin-only pills or LNG-IUD could be used for contraception in women with contraindications or intolerance for COCs.

Future studies should focus on the safety and potential longterm effects of COC use in well-defined populations with PCOS. A better understanding of these issues could be achieved by considering differences in the phenotypes of the syndrome as well as variations in the effectiveness and safety of different COCs.

\section{CONFLICTS OF INTEREST}

No potential conflict of interest relevant to this article was reported.

\section{ORCID}

Seda Hanife Oguz https://orcid.org/0000-0002-7781-944X

Bulent Okan Yildiz https://orcid.org/0000-0003-1797-7662

\section{REFERENCES}

1. Bozdag G, Mumusoglu S, Zengin D, Karabulut E, Yildiz BO. The prevalence and phenotypic features of polycystic ovary syndrome: a systematic review and meta-analysis. Hum Reprod 2016;31:2841-55.

2. Pincus G, Garcia CR, Rock J, Paniagua M, Pendleton A, Laraque F, et al. Effectiveness of an oral contraceptive; effects of a progestin-estrogen combination upon fertility, menstrual phenomena, and health. Science 1959;130:81-3.

3. Golobof A, Kiley J. The current status of oral contraceptives: progress and recent innovations. Semin Reprod Med 2016;34:145-51.

4. Gerstman BB, Piper JM, Tomita DK, Ferguson WJ, Stadel BV, Lundin FE. Oral contraceptive estrogen dose and the risk of deep venous thromboembolic disease. Am J Epidemiol 1991;133:32-7.

5. De Leo V, Musacchio MC, Cappelli V, Piomboni P, Morgante G. Hormonal contraceptives: pharmacology tailored to women's health. Hum Reprod Update 2016;22:634-46.

6. Yildiz BO. Approach to the patient: contraception in women with polycystic ovary syndrome. J Clin Endocrinol Metab 2015;100:794-802.

7. Christin-Maitre S. History of oral contraceptive drugs and their use worldwide. Best Pract Res Clin Endocrinol Metab 2013;27:3-12. 
8. Goebelsmann U, Mashchak CA, Mishell DR Jr. Comparison of hepatic impact of oral and vaginal administration of ethinyl estradiol. Am J Obstet Gynecol 1985;151:868-77.

9. Sitruk-Ware R, Nath A. Characteristics and metabolic effects of estrogen and progestins contained in oral contraceptive pills. Best Pract Res Clin Endocrinol Metab 2013; 27:13-24.

10. Timmer CJ, Geurts TB. Bioequivalence assessment of three different estradiol formulations in postmenopausal women in an open, randomized, single-dose, 3-way cross-over study. Eur J Drug Metab Pharmacokinet 1999;24:47-53.

11. Sitruk-Ware R, Nath A. The use of newer progestins for contraception. Contraception 2010;82:410-7.

12. Schindler AE, Campagnoli C, Druckmann R, Huber J, Pasqualini JR, Schweppe KW, et al. Classification and pharmacology of progestins. Maturitas 2008;61:171-80.

13. Oelkers W, Foidart JM, Dombrovicz N, Welter A, Heithecker R. Effects of a new oral contraceptive containing an antimineralocorticoid progestogen, drospirenone, on the renin-aldosterone system, body weight, blood pressure, glucose tolerance, and lipid metabolism. J Clin Endocrinol Metab 1995;80:1816-21.

14. Hemrika DJ, Slaats EH, Kennedy JC, de Vries RoblesKorsen TJ, Schoemaker J. Pulsatile luteinizing hormone patterns in long term oral contraceptive users. J Clin Endocrinol Metab 1993;77:420-6.

15. Rivera R, Yacobson I, Grimes D. The mechanism of action of hormonal contraceptives and intrauterine contraceptive devices. Am J Obstet Gynecol 1999;181(5 Pt 1):1263-9.

16. Fraser IS, Romer T, Parke S, Zeun S, Mellinger U, Machlitt A, et al. Effective treatment of heavy and/or prolonged menstrual bleeding with an oral contraceptive containing estradiol valerate and dienogest: a randomized, doubleblind Phase III trial. Hum Reprod 2011;26:2698-708.

17. Klipping C, Duijkers I, Trummer D, Marr J. Suppression of ovarian activity with a drospirenone-containing oral contraceptive in a 24/4 regimen. Contraception 2008;78:16-25.

18. Teede HJ, Misso ML, Costello MF, Dokras A, Laven J, Moran L, et al. Recommendations from the international evidence-based guideline for the assessment and management of polycystic ovary syndrome. Fertil Steril 2018;110: 364-79.

19. Rojanasakul A, Sirimongkolkasem R, Piromsawasdi S, Sumavong V, Chailurkit L, Chaturachinda K. Effects of combined ethinylestradiol and desogestrel on hormone profiles and sex hormone binding globulin in women with polycystic ovarian disease. Contraception 1987;36:633-40.

20. Stegeman BH, Raps M, Helmerhorst FM, Vos HL, van Vliet HA, Rosendaal FR, et al. Effect of ethinylestradiol dose and progestagen in combined oral contraceptives on plasma sex hormone-binding globulin levels in premenopausal women. J Thromb Haemost 2013;11:203-5.

21. Martin KA, Anderson RR, Chang RJ, Ehrmann DA, Lobo RA, Murad MH, et al. Evaluation and treatment of hirsutism in premenopausal women: an Endocrine Society clinical practice guideline. J Clin Endocrinol Metab 2018;103: 1233-57.

22. Fauser BC, Tarlatzis BC, Rebar RW, Legro RS, Balen AH, Lobo R, et al. Consensus on women's health aspects of polycystic ovary syndrome (PCOS): the Amsterdam ESHRE/ASRM-Sponsored 3rd PCOS Consensus Workshop Group. Fertil Steril 2012;97:28-38.

23. Legro RS, Arslanian SA, Ehrmann DA, Hoeger KM, Mu$\operatorname{rad} \mathrm{MH}$, Pasquali R, et al. Diagnosis and treatment of polycystic ovary syndrome: an Endocrine Society clinical practice guideline. J Clin Endocrinol Metab 2013;98:4565-92.

24. Conway G, Dewailly D, Diamanti-Kandarakis E, EscobarMorreale HF, Franks S, Gambineri A, et al. The polycystic ovary syndrome: a position statement from the European Society of Endocrinology. Eur J Endocrinol 2014;171:P1-29.

25. Barrionuevo P, Nabhan M, Altayar O, Wang Z, Erwin PJ, Asi N, et al. Treatment options for hirsutism: a systematic review and network meta-analysis. J Clin Endocrinol Metab 2018;103:1258-64.

26. Bahamondes L, Valeria Bahamondes M, Shulman LP. Noncontraceptive benefits of hormonal and intrauterine reversible contraceptive methods. Hum Reprod Update 2015;21: 640-51.

27. ESHRE Capri Workshop Group. Noncontraceptive health benefits of combined oral contraception. Hum Reprod Update 2005;11:513-25.

28. Jensen JT, Parke S, Mellinger U, Machlitt A, Fraser IS. Effective treatment of heavy menstrual bleeding with estradiol valerate and dienogest: a randomized controlled trial. Obstet Gynecol 2011;117:777-87.

29. Vlahos N, Vlachos A, Triantafyllidou O, Vitoratos N, Creatsas G. Continuous versus cyclic use of oral contraceptives after surgery for symptomatic endometriosis: a prospective cohort study. Fertil Steril 2013;100:1337-42.

30. Zorbas KA, Economopoulos KP, Vlahos NF. Continuous versus cyclic oral contraceptives for the treatment of endometriosis: a systematic review. Arch Gynecol Obstet 2015; 
292:37-43.

31. Schultheis P, Montoya MN, Zhao Q, Archer J, Madden T, Peipert JF. Contraception and ectopic pregnancy risk: a prospective observational analysis. Am J Obstet Gynecol 2021;224:228-9.

32. Burkman R, Schlesselman JJ, Zieman M. Safety concerns and health benefits associated with oral contraception. Am J Obstet Gynecol 2004;190(4 Suppl):S5-22.

33. Li C, Zhao WH, Meng CX, Ping H, Qin GJ, Cao SJ, et al. Contraceptive use and the risk of ectopic pregnancy: a multi-center case-control study. PLoS One 2014;9:e115031.

34. Lanza di Scalea T, Pearlstein T. Premenstrual dysphoric disorder. Med Clin North Am 2019;103:613-28.

35. Pearlstein TB, Bachmann GA, Zacur HA, Yonkers KA. Treatment of premenstrual dysphoric disorder with a new drospirenone-containing oral contraceptive formulation. Contraception 2005;72:414-21.

36. Michels KA, Pfeiffer RM, Brinton LA, Trabert B. Modification of the associations between duration of oral contraceptive use and ovarian, endometrial, breast, and colorectal cancers. JAMA Oncol 2018;4:516-21.

37. Karlsson T, Johansson T, Hoglund J, Ek WE, Johansson A. Time-dependent effects of oral contraceptive use on breast, ovarian and endometrial cancers. Cancer Res 2021;81: 1153-62.

38. Iversen L, Sivasubramaniam S, Lee AJ, Fielding S, Hannaford PC. Lifetime cancer risk and combined oral contraceptives: the Royal College of General Practitioners' Oral Contraception Study. Am J Obstet Gynecol 2017;216:580.

39. World Health Organization. Medical eligibility criteria for contraceptive use [Internet]. Geneva: WHO; 2015 [cited 2021 Mar 26]. Available from: https://www.who.int/publications/i/ item/9789241549158.

40. Curtis KM, Tepper NK, Jatlaoui TC, Berry-Bibee E, Horton LG, Zapata LB, et al. U.S. medical eligibility criteria for contraceptive use, 2016. MMWR Recomm Rep 2016;65:1103.

41. Heinemann LA, Dinger JC. Range of published estimates of venous thromboembolism incidence in young women. Contraception 2007;75:328-36.

42. Dragoman MV, Tepper NK, Fu R, Curtis KM, Chou R, Gaffield ME. A systematic review and meta-analysis of venous thrombosis risk among users of combined oral contraception. Int J Gynaecol Obstet 2018;141:287-94.

43. Kourlaba G, Relakis J, Kontodimas S, Holm MV, Maniadakis N. A systematic review and meta-analysis of the epide- miology and burden of venous thromboembolism among pregnant women. Int J Gynaecol Obstet 2016;132:4-10.

44. Barsoum MK, Heit JA, Ashrani AA, Leibson CL, Petterson TM, Bailey KR. Is progestin an independent risk factor for incident venous thromboembolism?: a population-based case-control study. Thromb Res 2010;126:373-8.

45. de Bastos M, Stegeman BH, Rosendaal FR, Van Hylckama Vlieg A, Helmerhorst FM, Stijnen T, et al. Combined oral contraceptives: venous thrombosis. Cochrane Database Syst Rev 2014;3:CD010813.

46. Practice Committee of the American Society for Reproductive Medicine; Practice Committee of the American Society for Reproductive Medicine. Combined hormonal contraception and the risk of venous thromboembolism: a guideline. Fertil Steril 2017;107:43-51.

47. van Hylckama Vlieg A, Helmerhorst FM, Vandenbroucke JP, Doggen CJ, Rosendaal FR. The venous thrombotic risk of oral contraceptives, effects of oestrogen dose and progestogen type: results of the MEGA case-control study. BMJ 2009;339:b2921.

48. Oedingen C, Scholz S, Razum O. Systematic review and meta-analysis of the association of combined oral contraceptives on the risk of venous thromboembolism: the role of the progestogen type and estrogen dose. Thromb Res 2018;165: $68-78$.

49. Dinger J, Do Minh T, Heinemann K. Impact of estrogen type on cardiovascular safety of combined oral contraceptives. Contraception 2016;94:328-39.

50. Manneras-Holm L, Baghaei F, Holm G, Janson PO, Ohlsson $\mathrm{C}$, Lonn $\mathrm{M}$, et al. Coagulation and fibrinolytic disturbances in women with polycystic ovary syndrome. J Clin Endocrinol Metab 2011;96:1068-76.

51. Yildiz BO, Haznedaroglu IC, Kirazli S, Bayraktar M. Global fibrinolytic capacity is decreased in polycystic ovary syndrome, suggesting a prothrombotic state. J Clin Endocrinol Metab 2002;87:3871-5.

52. Okoroh EM, Hooper WC, Atrash HK, Yusuf HR, Boulet SL. Is polycystic ovary syndrome another risk factor for venous thromboembolism? United States, 2003-2008. Am J Obstet Gynecol 2012;207:377.

53. Gariani K, Hugon-Rodin J, Philippe J, Righini M, Blondon M. Association between polycystic ovary syndrome and venous thromboembolism: a systematic review and metaanalysis. Thromb Res 2020;185:102-8.

54. Luque-Ramirez M, Ortiz-Flores AE, Nattero-Chavez L, Escobar-Morreale HF. A safety evaluation of current medica- 
tions for adult women with the polycystic ovarian syndrome not pursuing pregnancy. Expert Opin Drug Saf 2020; 19:1559-76.

55. Kasapoglu T. Do oral contraceptive pills really protect against venous thromboembolism, especially in polycystic ovary syndrome patients?: prescription bias or not? Am J Obstet Gynecol 2013;209:73-4.

56. Roach RE, Helmerhorst FM, Lijfering WM, Stijnen T, Algra A, Dekkers OM. Combined oral contraceptives: the risk of myocardial infarction and ischemic stroke. Cochrane Database Syst Rev 2015;8:CD011054.

57. Lidegaard O, Lokkegaard E, Jensen A, Skovlund CW, Keiding N. Thrombotic stroke and myocardial infarction with hormonal contraception. N Engl J Med 2012;366: 2257-66.

58. Helvaci N, Yildiz BO. Polycystic ovary syndrome and aging: health implications after menopause. Maturitas 2020; 139:12-9.

59. Wekker V, van Dammen L, Koning A, Heida KY, Painter $\mathrm{RC}$, Limpens J, et al. Long-term cardiometabolic disease risk in women with PCOS: a systematic review and metaanalysis. Hum Reprod Update 2020;26:942-60.

60. Gunning MN, Fauser BC. Are women with polycystic ovary syndrome at increased cardiovascular disease risk later in life? Climacteric 2017;20:222-7.

61. Schmidt J, Landin-Wilhelmsen K, Brannstrom M, Dahlgren E. Cardiovascular disease and risk factors in PCOS women of postmenopausal age: a 21-year controlled follow-up study. J Clin Endocrinol Metab 2011;96:3794-803.

62. Merz CN, Shaw LJ, Azziz R, Stanczyk FZ, Sopko G, Braunstein GD, et al. Cardiovascular disease and 10-year mortality in postmenopausal women with clinical features of polycystic ovary syndrome. J Womens Health (Larchmt) 2016;25:875-81.

63. Meun C, Franco OH, Dhana K, Jaspers L, Muka T, Louwers $\mathrm{Y}$, et al. High androgens in postmenopausal women and the risk for atherosclerosis and cardiovascular disease: the Rotterdam study. J Clin Endocrinol Metab 2018;103:162230.

64. Zhou Y, Wang X, Jiang Y, Ma H, Chen L, Lai C, et al. Association between polycystic ovary syndrome and the risk of stroke and all-cause mortality: insights from a metaanalysis. Gynecol Endocrinol 2017;33:904-10.

65. Glintborg D, Rubin KH, Nybo M, Abrahamsen B, Andersen M. Cardiovascular disease in a nationwide population of Danish women with polycystic ovary syndrome. Cardio- vasc Diabetol 2018;17:37.

66. Merz CN, Johnson BD, Berga S, Braunstein G, Reis SE, Bittner V, et al. Past oral contraceptive use and angiographic coronary artery disease in postmenopausal women: data from the National Heart, Lung, and Blood Institute-sponsored Women's Ischemia Syndrome Evaluation. Fertil Steril 2006;85:1425-31.

67. Forslund M, Landin-Wilhelmsen K, Trimpou P, Schmidt J, Brannstrom M, Dahlgren E. Type 2 diabetes mellitus in women with polycystic ovary syndrome during a 24-year period: importance of obesity and abdominal fat distribution. Hum Reprod Open 2020;2020:hoz042.

68. Rubin KH, Glintborg D, Nybo M, Abrahamsen B, Andersen M. Development and risk factors of type 2 diabetes in a nationwide population of women with polycystic ovary syndrome. J Clin Endocrinol Metab 2017;102:3848-57.

69. Troisi RJ, Cowie CC, Harris MI. Oral contraceptive use and glucose metabolism in a national sample of women in the United States. Am J Obstet Gynecol 2000;183:389-95.

70. Lopez LM, Grimes DA, Schulz KF. Steroidal contraceptives: effect on carbohydrate metabolism in women without diabetes mellitus. Cochrane Database Syst Rev 2019;11: CD006133.

71. Halperin IJ, Kumar SS, Stroup DF, Laredo SE. The association between the combined oral contraceptive pill and insulin resistance, dysglycemia and dyslipidemia in women with polycystic ovary syndrome: a systematic review and meta-analysis of observational studies. Hum Reprod 2011; 26:191-201.

72. Luque-Ramirez M, Nattero-Chavez L, Ortiz Flores AE, Escobar-Morreale HF. Combined oral contraceptives and/or antiandrogens versus insulin sensitizers for polycystic ovary syndrome: a systematic review and meta-analysis. Hum Reprod Update 2018;24:225-41.

73. Behboudi-Gandevani S, Ramezani Tehrani F, Hosseinpanah F, Khalili D, Cheraghi L, Kazemijaliseh H, et al. Cardiometabolic risks in polycystic ovary syndrome: long-term population-based follow-up study. Fertil Steril 2018;110: 1377-86.

74. Yildiz BO, Bozdag G, Yapici Z, Esinler I, Yarali H. Prevalence, phenotype and cardiometabolic risk of polycystic ovary syndrome under different diagnostic criteria. Hum Reprod 2012;27:3067-73.

75. Moran LJ, Misso ML, Wild RA, Norman RJ. Impaired glucose tolerance, type 2 diabetes and metabolic syndrome in polycystic ovary syndrome: a systematic review and meta-

Copyright (C) 2021 Korean Endocrine Society 
analysis. Hum Reprod Update 2010;16:347-63.

76. Sanchez-Garrido MA, Tena-Sempere M. Metabolic dysfunction in polycystic ovary syndrome: pathogenic role of androgen excess and potential therapeutic strategies. Mol Metab 2020;35:100937.

77. Cagnacci A, Paoletti AM, Renzi A, Orru M, Pilloni M, Melis GB, et al. Glucose metabolism and insulin resistance in women with polycystic ovary syndrome during therapy with oral contraceptives containing cyproterone acetate or desogestrel. J Clin Endocrinol Metab 2003;88:3621-5.

78. Wang A, Mo T, Li Q, Shen C, Liu M. The effectiveness of metformin, oral contraceptives, and lifestyle modification in improving the metabolism of overweight women with polycystic ovary syndrome: a network meta-analysis. Endocrine 2019;64:220-32.

79. Amiri M, Ramezani Tehrani F, Nahidi F, Kabir A, Azizi F, Carmina E. Effects of oral contraceptives on metabolic profile in women with polycystic ovary syndrome: a meta-analysis comparing products containing cyproterone acetate with third generation progestins. Metabolism 2017;73:22-35.

80. Amiri M, Nahidi F, Bidhendi-Yarandi R, Khalili D, Tohidi M, Ramezani Tehrani F. A comparison of the effects of oral contraceptives on the clinical and biochemical manifestations of polycystic ovary syndrome: a crossover randomized controlled trial. Hum Reprod 2020;35:175-86.

81. Li L, Zhang R, Zeng J, Ke H, Peng X, Huang L, et al. Effectiveness and safety assessment of drospirenone/ethinyl estradiol tablet in treatment of PCOS patients: a single center, prospective, observational study. BMC Womens Health 2020;20:39.

82. Fields EL, Trent ME. Treatment considerations for the cardiometabolic signs of polycystic ovary syndrome: a review of the literature since the 2013 Endocrine Society clinical practice guidelines. JAMA Pediatr 2016;170:502-7.

83. Hirschberg AL, Bystrom B, Carlstrom K, von Schoultz B. Reduced serum cholecystokinin and increase in body fat during oral contraception. Contraception 1996;53:109-13.

84. Arusoglu G, Koksal G, Cinar N, Tapan S, Aksoy DY, Yildiz BO. Basal and meal-stimulated ghrelin, PYY, CCK levels and satiety in lean women with polycystic ovary syndrome: effect of low-dose oral contraceptive. J Clin Endocrinol Metab 2013;98:4475-82.

85. Naessen S, Carlstrom K, Bystrom B, Pierre Y, Hirschberg AL. Effects of an antiandrogenic oral contraceptive on appetite and eating behavior in bulimic women. Psychoneuroendocrinology 2007;32:548-54.
86. Gallo MF, Lopez LM, Grimes DA, Carayon F, Schulz KF, Helmerhorst FM. Combination contraceptives: effects on weight. Cochrane Database Syst Rev 2014;1:CD003987.

87. Lindh I, Ellstrom AA, Milsom I. The long-term influence of combined oral contraceptives on body weight. Hum Reprod 2011;26:1917-24.

88. Aydin K, Cinar N, Aksoy DY, Bozdag G, Yildiz BO. Body composition in lean women with polycystic ovary syndrome: effect of ethinyl estradiol and drospirenone combination. Contraception 2013;87:358-62.

89. Pasquali R, Gambineri A, Anconetani B, Vicennati V, Colitta $\mathrm{D}$, Caramelli E, et al. The natural history of the metabolic syndrome in young women with the polycystic ovary syndrome and the effect of long-term oestrogen-progestagen treatment. Clin Endocrinol (Oxf) 1999;50:517-27.

90. Mes-Krowinkel MG, Louwers YV, Mulders AG, de Jong FH, Fauser BC, Laven JS. Influence of oral contraceptives on anthropomorphometric, endocrine, and metabolic profiles of anovulatory polycystic ovary syndrome patients. Fertil Steril 2014;101:1757-65.

91. Ibanez L, Diaz M, Sebastiani G, Marcos MV, Lopez-Bermejo A, de Zegher F. Oral contraception vs insulin sensitization for 18 months in nonobese adolescents with androgen excess: posttreatment differences in C-reactive protein, intima-media thickness, visceral adiposity, insulin sensitivity, and menstrual regularity. J Clin Endocrinol Metab 2013; 98:E902-7.

92. Harmanci A, Cinar N, Bayraktar M, Yildiz BO. Oral contraceptive plus antiandrogen therapy and cardiometabolic risk in polycystic ovary syndrome. Clin Endocrinol (Oxf) 2013; 78:120-5.

93. Shufelt C, LeVee A. Hormonal contraception in women with hypertension. JAMA 2020;324:1451-2.

94. Skovlund CW, Morch LS, Kessing LV, Lidegaard O. Association of hormonal contraception with depression. JAMA Psychiatry 2016;73:1154-62.

95. Schaffir J, Worly BL, Gur TL. Combined hormonal contraception and its effects on mood: a critical review. Eur J Contracept Reprod Health Care 2016;21:347-55.

96. Fruzzetti F, Fidecicchi T. Hormonal contraception and depression: updated evidence and implications in clinical practice. Clin Drug Investig 2020;40:1097-106.

97. Lundin C, Wikman A, Bixo M, Gemzell-Danielsson K, Sundstrom Poromaa I. Towards individualised contraceptive counselling: clinical and reproductive factors associated with self-reported hormonal contraceptive-induced ad- 
verse mood symptoms. BMJ Sex Reprod Health 2021 Jan 15 [Epub]. https://doi.org/10.1136/bmjsrh-2020-200658.

98. Cooney LG, Lee I, Sammel MD, Dokras A. High prevalence of moderate andcsevere depressive and anxiety symptoms in polycystic ovary syndrome: acsystematic review and meta-analysis. Hum Reprod 2017;32:1075-91.

99. Cinar N, Harmanci A, Demir B, Yildiz BO. Effect of an oral contraceptive on emotional distress, anxiety and depression of women with polycystic ovary syndrome: a prospective study. Hum Reprod 2012;27:1840-5.

100. Edwards AC, Lonn SL, Crump C, Moscicki EK, Sundquist J, Kendler KS, et al. Oral contraceptive use and risk of suicidal behavior among young women. Psychol Med 2020 Oct 21 [Epub]. https://doi.org/10.1017/S0033291720003475.

101. Dokras A, Sarwer DB, Allison KC, Milman L, Kris-Etherton PM, Kunselman AR, et al. Weight loss and lowering androgens predict improvements in health-related quality of life in women with PCOS. J Clin Endocrinol Metab 2016; 101:2966-74.

102. Yilmaz B, Yildiz BO. Endocrinology of hirsutism: from androgens to androgen excess disorders. Front Horm Res 2019;53:108-19.

103. Curtis KM, Jatlaoui TC, Tepper NK, Zapata LB, Horton LG, Jamieson DJ, et al. U.S. selected practice recommendations for contraceptive use, 2016. MMWR Recomm Rep 2016;65:1-66.

104. ACOG Practice Bulletin No. 206: use of hormonal contraception in women with coexisting medical conditions. Obstet Gynecol 2019;133:e128-50.

105. Coquoz A, Gruetter C, Stute P. Impact of micronized progesterone on body weight, body mass index, and glucose metabolism: a systematic review. Climacteric 2019;22:14861.

106. Kayikcioglu F, Gunes M, Ozdegirmenci O, Haberal A. Effects of levonorgestrel-releasing intrauterine system on glucose and lipid metabolism: a 1-year follow-up study. Contraception 2006;73:528-31.

107. Morin-Papunen L, Martikainen H, McCarthy MI, Franks S, Sovio U, Hartikainen AL, et al. Comparison of metabolic and inflammatory outcomes in women who used oral contraceptives and the levonorgestrel-releasing intrauterine device in a general population. Am J Obstet Gynecol 2008; 199:529.

108. Kivela A, Ruuskanen M, Agren U, Dieben T. The effects of two progrestogen-only pills containing either desogestrel (75 microgram/day) or levonorgestrel (30 microgram/day) on carbohydrate metabolism and adrenal and thyroid function. Eur J Contracept Reprod Health Care 2001;6:71-7.

109. Oderich CL, Wender MC, Lubianca JN, Santos LM, de Mello GC. Impact of etonogestrel-releasing implant and copper intrauterine device on carbohydrate metabolism: a comparative study. Contraception 2012;85:173-6.

110. Villas-Boas J, Vilodre LC, Malerba H, Pontremoli Salcedo M, Foresti Jimenez M, El Beitune P. Metabolic safety of the etonogestrel contraceptive implant in healthy women over a 3-year period. Eur J Obstet Gynecol Reprod Biol 2016;202:51-4.

111. Biswas A, Viegas OA, Coeling Bennink HJ, Korver T, Ratnam SS. Implanon contraceptive implants: effects on carbohydrate metabolism. Contraception 2001;63:137-41.

112. Centers for Disease Control and Prevention. Table C1. Classifications for progestin-only contraceptives [Internet]. Atlanta: CDC; 2020 [cited 2021 Mar 26]. Available from: https://www.cdc.gov/reproductivehealth/contraception/ mmwr/mec/appendixc_tablec1.html.

113. Grimes DA, Lopez LM, O’Brien PA, Raymond EG. Progestin-only pills for contraception. Cochrane Database Syst Rev 2013;11:CD007541.

114. Palacios S, Colli E, Regidor PA. Efficacy and cardiovascular safety of the new estrogen-free contraceptive pill containing $4 \mathrm{mg}$ drospirenone alone in a $24 / 4$ regime. BMC Womens Health 2020;20:218.

115. Zigler RE, McNicholas C. Unscheduled vaginal bleeding with progestin-only contraceptive use. Am J Obstet Gynecol 2017;216:443-50.

116. Hickey M, Higham JM, Fraser I. Progestogens with or without oestrogen for irregular uterine bleeding associated with anovulation. Cochrane Database Syst Rev 2012;9: CD001895.

117. Bagis T, Gokcel A, Zeyneloglu HB, Tarim E, Kilicdag EB, Haydardedeoglu B. The effects of short-term medroxyprogesterone acetate and micronized progesterone on glucose metabolism and lipid profiles in patients with polycystic ovary syndrome: a prospective randomized study. J Clin Endocrinol Metab 2002;87:4536-40.

118. Livadas S, Boutzios G, Economou F, Alexandraki K, Xyrafis X, Christou M, et al. The effect of oral micronized progesterone on hormonal and metabolic parameters in anovulatory patients with polycystic ovary syndrome. Fertil Steril 2010;94:242-6.

119. Barkfeldt J, Virkkunen A, Dieben T. The effects of two progestogen-only pills containing either desogestrel (75 mi- 
crog/day) or levonorgestrel (30 microg/day) on lipid metabolism. Contraception 2001;64:295-9.

120. Clark MK, Dillon JS, Sowers M, Nichols S. Weight, fat mass, and central distribution of fat increase when women use depot-medroxyprogesterone acetate for contraception. Int J Obes (Lond) 2005;29:1252-8.

121. Clark MK, Sowers MR, Nichols S, Levy B. Bone mineral density changes over two years in first-time users of depot medroxyprogesterone acetate. Fertil Steril 2004;82:1580-6.

122. Meyer C, Talbot M, Teede H. Effect of implanon on insulin resistance in women with polycystic ovary syndrome. Aust N Z J Obstet Gynaecol 2005;45:155-8.

123. Luukkainen T, Lahteenmaki P, Toivonen J. Levonorgestrelreleasing intrauterine device. Ann Med 1990;22:85-90.

124. Trussell J, Lalla AM, Doan QV, Reyes E, Pinto L, Gricar J. Cost effectiveness of contraceptives in the United States. Contraception 2009;79:5-14.

125. Mittermeier T, Farrant C, Wise MR. Levonorgestrel-releasing intrauterine system for endometrial hyperplasia. Cochrane Database Syst Rev 2020;9:CD012658.

126. Seeber B, Ziehr SC, Gschlieber A, Moser C, Mattle V, Seger C, et al. Quantitative levonorgestrel plasma level measurements in patients with regular and prolonged use of the levonorgestrel-releasing intrauterine system. Contraception 2012;86:345-9.

127. Mandelbaum RS, Ciccone MA, Nusbaum DJ, Khoshchehreh M, Purswani H, Morocco EB, et al. Progestin therapy for obese women with complex atypical hyperplasia: levonorgestrel-releasing intrauterine device vs systemic therapy. Am J Obstet Gynecol 2020;223:103.

128. Lin M, Xu X, Wang Y, Hu Y, Zhao Y. Evaluation of a levonorgestrel-releasing intrauterine system for treating endo- metrial hyperplasia in patients with polycystic ovary syndrome. Gynecol Obstet Invest 2014;78:41-4.

129. Fambrini M, Bargelli G, Peruzzi E, Buccoliero AM, Pieralli A, Andersson KL, et al. Levonorgestrel-releasing intrauterine system alone as primary treatment in young women with early endometrial cancer: case report. J Minim Invasive Gynecol 2009;16:630-3.

130. Tanmahasamut $\mathrm{P}$, Wongwananuruk T. Challenging regimen for long-term conservative treatment of endometrial adenocarcinoma in young women: a case report and review of the literature. Case Rep Oncol 2010;3:380-5.

131. Bofill Rodriguez M, Lethaby A, Jordan V. Progestogen-releasing intrauterine systems for heavy menstrual bleeding. Cochrane Database Syst Rev 2020;6:CD002126.

132. Lethaby A, Wise MR, Weterings MA, Bofill Rodriguez M, Brown J. Combined hormonal contraceptives for heavy menstrual bleeding. Cochrane Database Syst Rev 2019;2: CD000154.

133. Petta CA, Ferriani RA, Abrao MS, Hassan D, Rosa E Silva JC, Podgaec S, et al. Randomized clinical trial of a levonorgestrel-releasing intrauterine system and a depot GnRH analogue for the treatment of chronic pelvic pain in women with endometriosis. Hum Reprod 2005;20:1993-8.

134. Barnett C, Moehner S, Do Minh T, Heinemann K. Perforation risk and intra-uterine devices: results of the EURASIUD 5-year extension study. Eur J Contracept Reprod Health Care 2017;22:424-8.

135. da Silva AV, de Melo AS, Barboza RP, de Paula Martins W, Ferriani RA, Vieira CS. Levonorgestrel-releasing intrauterine system for women with polycystic ovary syndrome: metabolic and clinical effects. Reprod Sci 2016;23:877-84. 